\title{
HARISH-CHANDRA AND HIS WORK
}

\author{
REBECCA A. HERB
}

I began to study representation theory while I was a graduate student at the University of Washington in the early seventies. At that time learning the theory of unitary representations of semisimple Lie groups primarily meant learning Harish-Chandra's work, and it was not an easy task. By that time Harish-Chandra had published over fifty papers, more than a thousand pages, on this subject. His most important papers tended to consist of one or two pages of introduction followed by fifty to a hundred pages of dense mathematics. I was lucky because my thesis advisor, Garth Warner, knew those papers well enough that instead of saying, "Read HarishChandra's papers," or "Read Discrete series. I," he said, "Read p. 302 of Discrete series. I." The good thing about Harish-Chandra's papers was that if you knew what you were looking for and where to start, everything was written down. There were no mistakes, and the notation was always the same. You might have to refer back to three or four of his earlier papers for results, or even definitions, but he told you exactly where to look. I started on p. 302 of Discrete series. I with Lemma 56 and worked my way backward and forward, picking up a lemma here and there from earlier sections of the paper and from earlier papers. By the time I really understood that page, I was ready to write the first part of my thesis. In the seventeen years since I finished my $\mathrm{PhD}$ thesis, I have kept coming back to Harish-Chandra's papers, gradually picking up more and more pieces as I have needed to learn them for my own work. I never knew Harish-Chandra well personally, although I was lucky enough to spend two years at the Institute for Advanced Study and attend his weekly lectures on work in progress, but he has always been my mathematical hero, so I would like to take this opportunity to introduce you to Harish-Chandra and his work. But before I say anything about Harish-Chandra himself, I would like

Received by the editors January 23, 1991.

1980 Mathematics Subject Classification (1985 Revision). Primary 22E46.

This paper is the written version of an AMS-MAA invited address, delivered at the 1991 Annual Meeting on January 16, 1991 in San Francisco, California. 
to give a brief introduction to the field of representation theory and harmonic analysis.

The theory of harmonic analysis on groups originated in the eighteenth century with the problem, motivated by physical considerations, of representing an arbitrary function by a trigonometric series. In its modern version, the theory of Fourier series can be formulated as follows. Let $\mathbf{R}$ denote the additive group of real numbers, $2 \pi \mathbf{Z}$ the subgroup of $\mathbf{R}$ consisting of all integer multiples of $2 \pi$, and let $\mathbf{T}=\mathbf{R} / 2 \pi \mathbf{Z}$ be the quotient group. Then functions on $\mathbf{T}$ can be thought of as functions on the interval $[0,2 \pi]$ or as $2 \pi$-periodic functions on the real line. Lebesgue measure on $[0,2 \pi]$ gives a measure on $T$ which we normalize to have total mass one. Thus

$$
\int_{\mathbf{T}} f(x) d x=\frac{1}{2 \pi} \int_{0}^{2 \pi} f(x) d x .
$$

Instead of expanding real-valued functions on $\mathbf{T}$ in terms of the functions $\sin n x, \cos n x, n=0,1,2, \ldots$, it is more convenient to expand complex-valued functions on $\mathbf{T}$ in terms of the complex exponential functions $e^{i n x}=\cos n x+i \sin n x, n \in \mathbf{Z}$.

Suppose $f: \mathbf{T} \rightarrow \mathbf{C}$ is an integrable function, that is $f \in L^{1}(\mathbf{T})$. Then for each integer $n$ we can define a Fourier coefficient

$$
\hat{f}(n)=\int_{\mathbf{T}} f(x) e^{-i n x} d x .
$$

We then attach to $f$ the Fourier series

$$
f \leftrightarrow \sum_{n=-\infty}^{+\infty} \hat{f}(n) e^{i n x}
$$

The question is, in what sense does the Fourier series represent the function $f$ ?

In two situations the answer is very nice. First, if $f$ is sufficiently smooth, for example if $f$ is infinitely differentiable, then the Fourier series of $f$ converges to $f$ uniformly and absolutely. Thus for all $f \in C^{\infty}(\mathbf{T}), x \in \mathbf{T}$,

$$
f(x)=\sum_{n \in \mathbf{Z}} \hat{f}(n) e^{i n x}
$$

Second, let $L^{2}(\mathbf{T})$ denote the complex vector space of all measureable functions $f$ such that

$$
\|f\|_{2}=\left(\int_{\mathbf{T}}|f(x)|^{2} d x\right)^{\frac{1}{2}}
$$


is finite. We define an inner product on $L^{2}(\mathbf{T})$ by

$$
\langle f, g\rangle=\int_{\mathbf{T}} f(x) \overline{g(x)} d x .
$$

Then $L^{2}(\mathbf{T})$ is a Hilbert space, and if we set $e_{n}(x)=e^{i n x}$, then $\left\{e_{n}\right\}_{n=-\infty}^{+\infty}$ is an orthonormal basis for $L^{2}(\mathbf{T})$. Further, the Fourier coefficient $\hat{f}(n)=\left\langle f, e_{n}\right\rangle$ so that the Fourier series of $f \in L^{2}(\mathbf{T})$ is just the expansion of $f$ in terms of this orthonormal basis. Thus the partial sums of the Fourier series converge to $f$ in the $L^{2}$ norm, and

$$
f=\sum \hat{f}(n) e_{n}
$$

can be taken as an equality of $L^{2}$ functions.

I would now like to turn from the theory of harmonic analysis to that of group representations. Originally, for example in Burnside's classic Theory of groups of finite order published in 1897 [Bu], a group is defined to be a group of transformations of some set, for example a group of permutations of a finite set, a group of symmetries of some geometric object, or a group of linear transformations of a vector space. Of course now we think of a group as an abstract object which can be represented as a group of transformations. Because I want to discuss the connection between group representations and harmonic analysis, I will discuss only unitary linear representations of topological groups.

We will assume that $G$ is a locally compact topological group. That is, $G$ is a Hausdorff, locally compact topological space with a group structure which is compatible with the topology in the sense that the group operations are continuous. Let $W$ be a Hilbert space, that is a finite or infinite-dimensional complex vector space with inner product $\langle$,$\rangle , which is complete with respect to the$ norm $\|w\|=\langle w, w\rangle^{1 / 2}, w \in W$, coming from the inner product. A linear operator $T: W \rightarrow W$ is called unitary if $T$ is onto and $\langle T v, T w\rangle=\langle v, w\rangle$ for all $v, w \in W$. Let $U(W)$ be the group of unitary linear operators on $W$ and let $\pi: G \rightarrow U(W)$ be a group homomorphism. We assume that $\pi$ is continuous in the sense that for every $v, w \in W$, the complex-valued function $x \mapsto\langle\pi(x) v, w\rangle$ is continuous on $G$. Then $\pi$ or $(\pi, W)$ is called a unitary representation of $G$.

Example 1. Suppose that $\operatorname{dim} W=1$. In this case $U(W)$ is isomorphic to $S^{1}=\{z \in \mathbf{C}:|z|=1\}$, and using this identification, 
$\pi: G \rightarrow S^{1}$ can be thought of as a continuous group homomorphism from $G$ to $S^{1}$. One-dimensional unitary representations are called unitary characters.

Example 2. Since $G$ is a locally compact topological group, there is a unique (up to constant factor) right Haar measure on $G$. The main property of Haar measure is that it is translation invariant. That is, for every $f \in L^{1}(G), g \in G$,

$$
\int_{G} f(x) d x=\int_{G} f(x g) d x .
$$

Let $L^{2}(G)$ be the Hilbert space of square-integrable functions on $G$ with respect to this measure and define

$$
[R(g) f](x)=f(x g), x, \quad g \in G, f \in L^{2}(G) .
$$

Since we use right Haar measure to define the inner product in $L^{2}(G)$, each operator $R(g), g \in G$, is unitary. Thus we have a group homomorphism

$$
R: G \rightarrow U\left(L^{2}(G)\right)
$$

of $G$ into the group of unitary operators on the Hilbert space $L^{2}(G)$. It is called the right regular representation of $G$.

Let $(\pi, W)$ be a unitary representation of $G$. A closed subspace $V \subset W$ is called invariant if $\pi(g) v \in V$ for all $g \in G, v \in$ $V$. In this case we obtain a unitary representation of $G$ on $V$ by restricting the operators $\pi(g), g \in G$, to $V$. Further, if $V$ is invariant, so is

$$
V^{\perp}=\{w \in W:\langle v, w\rangle=0 \text { for all } v \in V\} .
$$

Thus we can regard $(\pi, W)$ as the direct sum of representations of $G$ on $V$ and $V^{\perp}$. That is $W=V \oplus V^{\perp}$ is a decomposition of $W$ into simpler pieces. Now $(\pi, W)$ is called irreducible if it has no proper invariant closed subspaces. For example, if $W$ is one-dimensional, $(\pi, W)$ is irreducible since $W$ has no proper subspaces. If $G$ is abelian, these are the only irreducible representations. However nonabelian groups have higher dimensional, and even infinite-dimensional irreducible representations.

The two most important problems in representation theory are the following.

(1) Given a locally compact group $G$, find $\hat{G}$, the set of irreducible unitary representations. Of course there is a natural notion of two representations being "the same" and so we want a list of representations up to equivalence. 
(2) Given an arbitrary unitary representation of $G$, describe how to decompose it into irreducible constituents. The most important case here is the decomposition of the regular representation of $G$ on $L^{2}(G)$. This is $L^{2}$ harmonic analysis on $G$.

Example 3. Let $G=\mathbf{T}=\mathbf{R} / 2 \pi \mathbf{Z}$. $\mathbf{T}$ is an abelian group so $\hat{\mathbf{T}}$ is the set of unitary characters of $\mathbf{T}$. For each $n \in \mathbf{Z}$, the function $e_{n}(x)=e^{i n x}, x \in \mathbf{T}$, is a unitary character of $\mathbf{T}$. It is an advanced calculus exercise to prove that these are the only continuous homomorphisms of $\mathbf{T}$ into $S^{1}$. Thus $\hat{\mathbf{T}} \cong \mathbf{Z}$. For each $n \in \mathbf{Z}, \mathbf{C} e_{n} \subset L^{2}(\mathbf{T})$ is an invariant subspace of $L^{2}(\mathbf{T})$ and the $L^{2}$ theory of Fourier series says that

$$
L^{2}(\mathbf{T})=\bigoplus \sum_{n \in \mathbf{Z}} \mathbf{C} e_{n}
$$

is the decomposition of $L^{2}(\mathbf{T})$ into irreducible subspaces. Further, for each $n \in \mathbf{Z}$, the projection of $L^{2}(\mathbf{T})$ onto $\mathrm{C}_{n}$ is given by $f \mapsto \hat{f}(n) e_{n}, f \in L^{2}(\mathbf{T})$.

Example 4. Suppose $G$ is a compact group. Then every $(\pi, W) \in$ $\hat{G}$ is finite-dimensional. The dimension $d_{\pi}$ of $W$ is called the degree of $\pi$. We define a matrix coefficient of $(\pi, W) \in \hat{G}$ to be any function of the form $x \mapsto\langle\pi(x) v, w\rangle, v, w \in W$. These are all continuous functions by our continuity requirement on $\pi$, and $G$ is compact, so every matrix coefficient is in $L^{2}(G)$. Let $L^{2}(G)_{\pi}$ denote the subspace of $L^{2}(G)$ spanned by the matrix coefficients of $\pi \in \hat{G}$. It is an invariant subspace and the restriction of the regular representation to $L^{2}(G)_{\pi}$ is equivalent to $d_{\pi}$ copies of $\pi$. Functions in $L^{2}(G)_{\pi}$ are regarded as elementary functions because they transform in the simplest possible way with respect to translations by elements of the group. The Peter-Weyl theorem says that

$$
L^{2}(G)=\bigoplus \sum_{\pi \in \hat{G}} L^{2}(G)_{\pi}
$$

That is, $L^{2}(G)$ decomposes as the orthogonal direct sum of irreducible subspaces, and each $\pi \in \hat{G}$ occurs with multiplicity $d_{\pi}$. The analogue of the Fourier series for a function $f \in L^{2}(G)$ can now be defined as follows. The character of $\pi \in \hat{G}$ is the element of $L^{2}(G)_{\pi}$ defined by

$$
\Theta_{\pi}(x)=\operatorname{trace} \pi(x), \quad x \in G .
$$


Now the projection of $L^{2}(G)$ onto the subspace $L^{2}(G)_{\pi}$ is given by $f \mapsto f_{\pi}$ where

$$
f_{\pi}(x)=d_{\pi}\left(\Theta_{\pi} * f\right)(x)=d_{\pi} \int_{G} \Theta_{\pi}\left(x y^{-1}\right) f(y) d y .
$$

Thus we have an expansion of $f \in L^{2}(G)$ in terms of elementary functions,

$$
f=\sum_{\pi \in \hat{G}} d_{\pi}\left(\Theta_{\pi} * f\right), \quad f \in L^{2}(G) .
$$

This equality can be interpreted in the $L^{2}$ sense that the partial sums of the infinite series converge to $f$ in $L^{2}(G)$ or can be taken literally if $f$ is smooth enough that the series converges pointwise to $f$. It is called the Plancherel formula for $G$ and the measure that assigns to each $\pi \in \hat{G}$ the mass $d_{\pi}$ is called the Plancherel measure on $\hat{G}$. Thus the theory of characters of irreducible unitary representations of $G$ yields a theory of harmonic analysis on $G$.

Example 5. Let $G$ be any locally compact abelian group. Then all its irreducible unitary representations are one-dimensional, that is are unitary characters. However, if $G$ is noncompact, these characters are not $L^{2}$ functions since they have constant absolute value one. But $L^{2}(G)$ can still be decomposed in terms of these characters as a "direct integral" rather than as a direct sum. This generalizes the theory of the Fourier transform for functions on the real line. For $f \in L^{1}(G) \cap L^{2}(G)$ we can define Fourier coefficients

$$
\hat{f}(\pi)=\int_{G} f(x) \overline{\pi(x)} d x, \quad \pi \in \hat{G} .
$$

Further, the set $\hat{G}$ of unitary characters is itself a locally compact abelian group so it has a Haar measure. The Plancherel theorem says that $f \mapsto \hat{f}$ extends to an isometry of $L^{2}(G)$ onto $L^{2}(\hat{G})$. Just as before there is a Fourier inversion formula that says that if $f \in L^{2}(G)$ is sufficiently nice,

$$
f(g)=\int_{\hat{G}} \hat{f}(\pi) \pi(g) d \pi
$$

where $d \pi$ is the (suitably normalized) Haar measure on $\hat{G}$. Thus we can think of

$$
L^{2}(G)=\bigoplus \int_{\hat{G}} \mathbf{C} \pi d \pi
$$


Of course a one-dimensional representation is its own character, and it is easy to check that

$$
\hat{f}(\pi) \pi(g)=(\pi * f)(g)
$$

where the convolution is defined as in the compact case. Thus we can write

$$
f=\int_{\hat{G}}(\pi * f) d \pi
$$

as in the compact case. Thus the Plancherel measure on $\hat{G}$ is just the Haar measure and we can think of $L^{2}(G)$ as a direct integral of irreducible representations with respect to Haar measure.

I would now like to return to Harish-Chandra. The most complete reference for biographical information about Harish-Chandra, and my main source for the material that follows, is a memoir by R. P. Langlands [L]. A good introduction to Harish-Chandra's work on semisimple Lie groups is V.S. Varadarajan's introduction to Harish-Chandra's Collected Papers [H]. I would like to thank Professor Varadarajan for also making available to me an article on Harish-Chandra which will appear in the Journal of the Indian Mathematical Society [V].

Harish-Chandra was born in 1923 in northern India. His earliest papers were written in India in the early 1940s in the field of particle physics. Then, in 1945, he went to Cambridge, England, to work with Dirac. At this time the basic representation theory of abelian and compact groups was well understood. However, very little was known about nonabelian, noncompact groups. These groups occur as symmetry groups of quantum mechanical systems, and physicists, starting with E. Wigner in 1939 [W], were just beginning to study their infinite-dimensional unitary representations. Dirac realized that this was a wide open area, and suggested that Harish-Chandra study the infinite-dimensional unitary representations of the Lorentz group, $S O(1,3)$. He did this in his $\mathrm{PhD}$ thesis, completed in 1947. He then visited the Institute for Advanced Study in Princeton. Langlands's memoir includes the following comment by Harish-Chandra.

"Soon after coming to Princeton I became aware that my work on the Lorentz group was based on somewhat shaky arguments. I had naively manipulated unbounded operators without paying any attention to their domains of definition. I once complained to Dirac about the fact that my proofs were not rigorous and he replied, 'I am not interested in proofs but only in what nature 
does.' This remark confirmed my growing conviction that I did not have the mysterious sixth sense which one needs in order to succeed in physics and I soon decided to move over to mathematics."

Thus, although Harish-Chandra greatly admired the work of Dirac and other theoretical physicists, around 1950 he abandoned physics and began the rigorous mathematical study of the representation theory and harmonic analysis of semisimple Lie groups. He devoted the remainder of his life to this and related projects. He held a position at Columbia University from 1950-1963, and then from 1963 until his death in 1983 was a member of the Institute for Advanced Study.

I have tried to give you some idea of what representation theory and harmonic analysis are. But what is a semisimple Lie group? Although there is a more general definition, for simplicity we will consider only linear Lie groups, that is closed subgroups of $G L(n, \mathbf{R})$ or $G L(n, \mathbf{C})$, the groups of invertible $n \times n$ matrices with real or complex entries. On a Lie group $G$ therefore, in addition to the topological group structure, one has an analytic structure coming from the embedding of $G$ in the Euclidean space $\mathbf{R}^{n^{2}}$ or $\mathbf{C}^{n^{2}}$. Let $G$ be a connected, linear Lie group. $G$ is called reductive if it is stable under conjugate transpose, and is called semisimple if it is reductive, nonabelian, and has finite center. For example, $G L(n, \mathbf{C})$ is reductive, but not semisimple, since its center, the set of all scalar matrices, is isomorphic to $\mathbf{C}^{\times}$. $G=S L(n, \mathbf{C})=\{g \in G L(n, \mathbf{C}): \operatorname{det} g=1\}$ is semisimple since the condition det $g=1$ is stable under conjugate transpose, and the center of $G$, the set of all scalar matrices with determinant one, is isomorphic to the group of complex $n$th roots of unity.

The class of semisimple Lie groups contains many of the groups of interest to physics. In addition to Harish-Chandra's own work on the Lorentz group, the group $S L(2, \mathbf{R})$ of real two-by-two matrices with determinant one had been studied by Bargmann in 1947 [Ba], and the groups $S L(n, \mathrm{C})$ of $n \times n$ complex matrices with determinant one had been studied by Gelfand and Naimark in 1950 [GN]. Work in representation theory at that time either focused on specific classical groups, or was of a very general nature. Many mathematicians were optimistic that the tools of functional analysis and operator algebras were sufficient to solve the problems of representation theory for all locally compact topological groups. Thus Harish-Chandra's decision to study semisimple Lie 
groups as a class was very much against the prevailing fashion. In retrospect however, it was an excellent choice. First, the semisimple Lie groups have a detailed structure theory which had been worked out by Chevalley in his three volume Theory of Lie groups [C1, C2, C3]. Harish-Chandra was able to exploit this structure to obtain a beautiful and complete theory of harmonic analysis. Second, the semisimple groups occupy a central position in the theory of general Lie groups similar to that played by simple groups in finite group theory. Work by other mathematicians, for example G. Mackey in the 1950s [M1, M2] and M. Duflo in the early 1980s [D], has shown that many problems in the representation theory of general Lie groups can be understood once the semisimple case is known.

Recall as above that the basic problems of harmonic analysis for a group $G$ are to find $\hat{G}$, the irreducible unitary representations, and then to find the Plancherel measure on $\hat{G}$ that gives the decomposition of the regular representation of $G$ on $L^{2}(G)$ into irreducible constituents. For compact and abelian groups this is carried out via the theory of characters. This is possible because the irreducible unitary representations involved are finitedimensional. However, for a noncompact simple Lie group, the only finite-dimensional irreducible unitary representation is the trivial unitary character $\pi(x)=1$ for all $x \in G$. However, semisimple Lie groups have many infinite-dimensional irreducible unitary representations which provide a theory of harmonic analysis.

Thus the first problem in generalizing the theory for compact and abelian groups to a semisimple Lie group $G$ is to define the character of an infinite-dimensional irreducible unitary representation $\pi \in \hat{G}$. In this case the unitary operators $\pi(x), x \in G$, do not have a well-defined trace since they have infinitely many eigenvalues all of absolute value one. However the character can be defined as a distribution as follows. Given a smooth, compactly supported function $f \in C_{c}^{\infty}(G)$, define

$$
\pi(f)=\int_{G} f(x) \pi(x) d x .
$$

Thus $\pi(f)$ is an operator on the representation space $W$ of $\pi$. It turns out that $\pi(f)$ is of trace class and that

$$
f \mapsto \Theta_{\pi}(f)=\operatorname{trace} \pi(f)
$$


is a distribution on $G$, that is a continuous linear functional on $C_{c}^{\infty}(G)$. This distribution is called the character of $\pi$. Now we say that $\mu$ is the Plancherel measure on $\hat{G}$ if

$$
f(x)=\int_{\hat{G}} \Theta_{\pi}(R(x) f) d \mu(\pi), \quad x \in G, f \in C_{c}^{\infty}(G) .
$$

Here $\Theta_{\pi}(R(x) f)$, the distribution $\Theta_{\pi}$ evaluated at the function $R(x) f$, plays the same role as the convolution $\left(\Theta_{\pi} * f\right)(x)$ of functions in the compact case. Since $f(x)=(R(x) f)(e)$, it is equivalent to write

$$
f(e)=\int_{\hat{G}} \Theta_{\pi}(f) d \mu(\pi), \quad f \in C_{c}^{\infty}(G) .
$$

Thus Harish-Chandra thought of the Plancherel theorem as an expansion of the Dirac delta distribution, $f \rightarrow f(e)$, in terms of the distribution characters of the irreducible unitary representations.

Before I say more about Harish-Chandra's work I would like to give a brief description of the Plancherel formula for $S L(2, \mathbf{R})$. It is the prototype semisimple Lie group, and most of the interesting features of the general case already occur here.

Example 6. Let

$$
G=S L(2, \mathbf{R})=\left\{\left(\begin{array}{ll}
a & b \\
c & d
\end{array}\right): a, b, c, d \in \mathbf{R}, a d-b c=1\right\}
$$

be the group of $2 \times 2$ matrices with real entries and determinant one. We parameterize irreducible unitary representations of $G$ by unitary characters of certain abelian subgroups called Cartan subgroups. These are maximal abelian subgroups consisting of elements which are semisimple, that is can be diagonalized over the field of complex numbers. In this case there are two conjugacy classes of Cartan subgroups.

One is the group

$$
A=\left\{d(a)=\left(\begin{array}{cc}
a & 0 \\
0 & a^{-1}
\end{array}\right): a \in \mathbf{R}, a \neq 0\right\}
$$

of diagonal matrices in $G$. Since $A \cong \mathbf{R}^{\times}$, the multiplicative group of nonzero real numbers, the unitary characters of $A$ are given by the set of all

$$
\sigma(\nu: \varepsilon)(d(a))=\left(\frac{a}{|a|}\right)^{\varepsilon}|a|^{i \nu}, \nu \in \mathbf{R}, \varepsilon \in\{0,1\} .
$$

Let

$$
P=\left\{p(a, b)=\left(\begin{array}{cc}
a & b \\
0 & a^{-1}
\end{array}\right): a, b \in \mathbf{R}, a \neq 0\right\}
$$


be the group of upper triangular matrices in $G$. Any $\sigma \in \hat{A}$ extends to a unitary character of $P$ by

$$
\sigma(p(a, b))=\sigma(d(a)) .
$$

Now there is a process called unitary induction which starts with a unitary representation $\sigma$ of the subgroup $P$ and produces a unitary representation $\pi=\operatorname{Ind}_{P}^{G}(\sigma)$ of the group $G$. Write

$$
\pi(A: \nu: \varepsilon)=\operatorname{Ind}_{P}^{G}(\sigma(\nu: \varepsilon)) .
$$

These representations are called the principal series of $G$. They are all unitary infinite-dimensional representations and are irreducible except when $\nu=0$ and $\varepsilon=1$, which decomposes as the sum of two irreducible representations. Write $\Theta(A: \nu: \varepsilon)$ for the distribution character of $\pi(A: \nu: \varepsilon)$.

The other Cartan subgroup is the group

$$
T=\left\{\left(\begin{array}{cc}
\cos \theta & \sin \theta \\
-\sin \theta & \cos \theta
\end{array}\right): \theta \in \mathbf{T}\right\} \text {. }
$$

In this case $\hat{T} \cong \mathbf{Z}$, and each $n \in \mathbf{Z}, n \neq 0$, parameterizes an irreducible unitary representation $\pi(T: n)$ of $G$. In this case there is no simple induction procedure for producing $\pi(T: n)$. These representations are called the discrete series of $G$. For each $n \neq 0$, the matrix coefficients of $\pi(T: n)$ are square-integrable, so that they span an invariant subspace of $L^{2}(G)$. Write $\Theta(T: n)$ for the distribution character of $\pi(T: n)$.

The Plancherel theorem for $G$, proven by Harish-Chandra in 1952 , says that for every $f \in C_{c}^{\infty}(G)$, we have

$$
\begin{aligned}
f(e)= & \sum_{n \in \mathbf{Z}}|n| \Theta(T: n)(f) \\
& +\frac{1}{4} \int_{-\infty}^{\infty} \Theta(A: \nu: 0)(f) \nu \tanh \left(\frac{\pi \nu}{2}\right) d \nu \\
& +\frac{1}{4} \int_{-\infty}^{\infty} \Theta(A: \nu: 1)(f) \nu \operatorname{coth}\left(\frac{\pi \nu}{2}\right) d \nu .
\end{aligned}
$$

That is the Plancherel measure assigns to the discrete series representation $\pi(T: n)$ the point mass $|n|$. The weight $|n|$ is called the formal degree of $\pi(T: n)$ since it plays the same role as the degree of a finite-dimensional irreducible representation of a compact group. The Plancherel measure for the principal series $\pi(A: \nu: \varepsilon)$ is absolutely continuous with respect to Lebesgue measure $d \nu$ and 
is given by $(\nu / 4) \tanh (\pi \nu / 2) d \nu$ or $(\nu / 4) \operatorname{coth}(\pi \nu / 2) d \nu$ depending upon whether $\varepsilon=0$ or 1 . There are other irreducible unitary representations called the complementary series, but they have Plancherel measure zero, and so do not contribute to the harmonic analysis of $L^{2}(G)$.

Harish-Chandra's work on semisimple groups was directed specifically towards the goal of obtaining an explicit Plancherel formula. Because of this there were aspects of representation theory which he did not study. First, he did not attempt to obtain a complete list of irreducible unitary representations of semisimple Lie groups. This is in fact still an open problem. He only needed to produce those, called the tempered representations, which occur in the Plancherel formula. Of the exceptional representations which do not occur in the Plancherel formula Harish-Chandra said in 1965, "Selberg tells me that they are important in arithmetic, and I believe him because he is always right." He was interested in the philosophical question of why they exist, but had no motivation to study them himself. Second, for the Plancherel formula, the representations themselves are less important than their characters. Thus it is more important to understand the characters of the representations than it is to find an explicit realization of the representations as operators on Hilbert spaces.

Harish-Chandra first concentrated his attention on character theory. Although the characters of infinite-dimensional unitary representations must be defined as distributions, the known examples were given by integration against locally summable functions. By the early 1960s Harish-Chandra had proven that this was true for the character of any irreducible unitary representation of a semisimple Lie group. That is, given $\pi \in \hat{G}$, there is a function $T_{\pi}$ on $G$ which is integrable over any compact subset of $G$ and which satisfies

$$
\Theta_{\pi}(f)=\int_{G} f(x) T_{\pi}(x) d x
$$

for every $f \in C_{c}^{\infty}(G)$. Further, there is a dense open subset $G^{\prime}$ of $G$ such that $T_{\pi}$ is analytic on $G^{\prime}$. This is known as the regularity theorem. One of the main steps in the proof is the reduction to a similar theorem on the Lie algebra. On the Lie algebra he could use classical Fourier analysis to solve the problem. This was a technique that Harish-Chandra used in many situtations and can be thought of as linearizing problems on the group. 
As early as 1954 Harish-Chandra realized that every Cartan subgroup, that is maximal abelian subgroup, gives rise to a series of irreducible unitary reprentations and that compact Cartan subgroups should give the discrete series representations. He was able to construct these discrete series representations in some cases. However, even after years of effort, he was unable to find a construction which produced all discrete series representations. Finally, he realized that it was not necessary to construct the representations. It was enough to construct their characters as functions on the group, and this he was able to do. Basically, Harish-Chandra showed that discrete series representations are parameterized by characters of compact Cartan subgroups. In particular, a group has discrete series if and only if it has a compact Cartan subgroup. The discrete series character is uniquely determined by its restriction to the compact Cartan subgroup plus a growth condition. On the compact Cartan subgroup, the discrete series character has a formula completely analogous to the Weyl character formula for compact groups. In general, the character is given by a sum of exponential functions with integer coefficients, divided by a universal denominator. There are relations among the coefficients which determine them uniquely. This work was completed in two papers, Discrete series. I (1965) and Discrete series. II (1966), and was a real tour de force, the culmination of over a decade of intense effort. It was one of the most important and difficult steps in Harish-Chandra's study of semisimple Lie groups.

Once the discrete series was in place, Harish-Chandra was able to construct a series of representations corresponding to each conjugacy class of Cartan subgroups as follows. In general, Cartan subgroups of semisimple Lie groups have the form $H=T \times A$ where $T$ is compact, and $A \cong \mathbf{R}^{n}$ for some $n$. Associated to $H$ is a parabolic subgroup $P=M A N$. Here $M$ centralizes $A$, contains $T$, and is a group of the same general type as the original group $G$. Now if $(\sigma, V)$ is any irreducible unitary representation of $M$ and $\nu \in \hat{A}$ is a unitary character of $A$, then

$$
\sigma_{\nu}(\text { man })=\nu(a) \sigma(m), \quad m \in M, a \in A, n \in N,
$$

defines an irreducible unitary representation of $P=M A N$ on $V$. Define

$$
\pi(P: \sigma: \nu)=\operatorname{Ind}_{P}^{G}\left(\sigma_{\nu}\right)
$$

to be the induced representation. These representations of $G$ are unitary and generically irreducible. However, there is overlap 
between the different series. For example, if $H=T$ is compact so that $A=1$, then $P=M=G$ and no induction takes place. Every $\sigma \in \hat{G}$ would occur in this series. However, it is only the discrete series of $G$ which cannot be obtained by induction from proper parabolic subgroups. One of Harish-Chandra's conceptual breakthroughs was to realize that in general, if only the discrete series representations of $M$ are used in the above construction, then all tempered representations of $G$ are still obtained, and there is now no overlap between the different series. Thus the final picture is as follows. Recall that we started with a Cartan subgroup $H=T \times A$. Now $T$ is a compact Cartan subgroup of $M$, so unitary characters $\chi \in \hat{T}$ parameterize discrete series representations $\pi(M: \chi)$ of $M$. Thus the series of unitary representations corresponding to $H$ is parameterized by pairs $(\chi, \nu), \chi \in \hat{T}, \nu \in \hat{A}$. Write $\Theta(H: \chi: \nu)$ for the distribution character of the corresponding representation, $\pi(P: \sigma(M: \chi): \nu)$.

Once Harish-Chandra had these series of representations he set about proving the Plancherel formula. The proof is long and complicated, and appears in three lengthy papers, Harmonic analysis on real reductive groups. I, II, III, which were published in 1975 and 1976. One of the most important ingredients of the proof is a detailed asymptotic analysis of the matrix coefficients of the tempered representations. This analysis of the behavior at infinity of matrix coefficients comes from a study of the differential equations which they satisfy plus certain a priori estimates. The final answer, however, is very simple. The Plancherel formula has the following form. For $f \in C_{c}^{\infty}(G)$,

$$
f(e)=\sum_{H=T \times A} \sum_{\chi \in \hat{T}} d_{\chi} \int_{\hat{A}} \Theta(H: \chi: \nu)(f) \mu(H: \chi: \nu) d \nu .
$$

Here the first sum is over a set of representatives $H$ for conjugacy classes of Cartan subgroups of $G$. (There are only finitely many conjugacy classes.) For fixed $H=T \times A$ corresponding to $P=$ $M A N, d_{\chi}, \chi \in \hat{T}$, is the formal degree of the discrete series representation $\pi(M: \chi)$ of $M$, and $d \nu$ is Lebesgue measure on $\hat{A} \cong \mathbf{R}^{n}$. The Plancherel function $\mu(H: \chi: \nu)$ comes from the asymptotic analysis of the matrix coefficients of the representations $\pi(H: \chi: \nu)$ and has an explicit formula as a product of terms of the type that occur in the Plancherel formula for $S L(2, \mathbf{R})$. This product formula is the key ingredient in the proof, and ultimately allowed Harish-Chandra to reduce the problem to the case of rank 
one groups where he could prove the Plancherel formula directly as for the case of $S L(2, \mathbf{R})$.

Many parts of the proof proceed via induction on the dimension of the group $G$ and involve reducing from $G$ to the subgroups $M$ used above in defining the various series of representations. When $G$ is connected and semisimple, these subgroups $M$ are not quite connected or semisimple. In order to make the induction work Harish-Chandra had to work with a larger class of groups, now known as the reductive groups of Harish-Chandra class, which contains the connected, linear, semisimple groups and is closed under passing from $G$ to the subgroups $M$. Induction was in fact Harish-Chandra's preferred method of proof and he was a master of the technique. He compared induction to high finance. "If you don't borrow enough, you have cash flow problems. If you borrow too much, you can't pay the interest."

I have only described here the bare bones of Harish-Chandra's theory, namely the Plancherel measure itself. In fact, in the course of proving the Plancherel theorem, Harish-Chandra defined a Schwartz space of smooth, rapidly decreasing functions on $G$ which is analogous to the classical Schwartz space for the real line. In the classical situation one proves that a function is Schwartz class if and only if its Fourier transform is Schwartz class. In the case that $G$ is a semisimple Lie group, one can think of the collection of characters $\Theta(H: \chi: \nu)(f)$ as the Fourier transform of $f$. Harish-Chandra proved that the Schwartz functions $f$ which have a technical property called $K$-finiteness are characterized by the fact that their Fourier transforms $\Theta(H: \chi: \nu)(f)$ are classical Schwartz functions of the real variable $\nu$.

I would like to conclude with a few comments about HarishChandra and his mathematics.

Throughout most of his career Harish-Chandra focussed on a very specific problem - the harmonic analysis of semisimple groups, first semisimple Lie groups and then semisimple matrix groups over other fields such as the $p$-adic fields which play an important role in number theory. Although the problems Harish-Chandra focussed on were narrow, he used a wide range of techniques to solve them. He learned what he needed from the work of others, but primarily manufactured his own tools as the need arose. He had incredible power, both as an analyst and algebraist. He started with the structure theory from Chevalley's three volumes and honed it to obtain a very exact knowledge of these groups. Thus he was 
always able to give general as opposed to case by case proofs. In fact, many of us in the field used to speculate as to whether HarishChandra ever worked any examples apart from that of $S L(2, \mathbf{R})$. In his biography, Langlands specifically mentions one example in the late fifties when, contrary to custom, Harish-Chandra verified a result for the group $S L(3, \mathbf{R})$ by an explicit calculation. Langlands concludes, "This appears to have given him the necessary confidence that it was true in general."

Perhaps because the details of this work were so technical, Harish-Chandra liked to have a simple philosophy to guide his efforts. He says in the introduction to a survey paper, "Our entire approach to harmonic analysis on reductive groups is based on the philosophy of cusp forms." The term cusp form comes from the theory of automorphic forms and was used by Harish-Chandra as a name for the matrix coefficients of the discrete series representations which play a pivotal role in the harmonic analysis. The strong analogy between his work on real groups and the theory of automorphic forms from number theory led him to believe that whatever is true for a real reductive group is true for a $p$-adic reductive group. He called this the Lefschetz Principle because of its resemblance to the Lefschetz principle in algebraic geometry. It led him to a proof of the Plancherel formula in the $p$-adic context as well, although in this case he was unable to explicitly parameterize the discrete series. This unified theory for real and $p$-adic groups reinforced his belief that harmonic analysis on a semisimple group was a special thing. At the end of his 1972 lecture series at the Williamstown conference, he told a story which he attributed to Chevalley. The story relates to the time before Genesis when God and his faithful servant, the Devil, were preparing to create the universe. God gave the Devil pretty much a free hand in building things, but told him to keep off certain objects to which He Himself would attend. Chevalley's story was that semisimple groups were among the special items. Harish-Chandra added that he hoped that the Lefschetz principle was also on the special list.

Let me conclude by quoting Varadarajan's introduction to Harish-Chandra's Collected Papers $[\mathrm{H}]$. Harish-Chandra "survives in his work, which is a faithful reflection of his personality-lofty, intense, uncompromising." 


\section{REFERENCES}

[Ba] V. Bargmann, Irreducible unitary representations of the Lorentz group, Ann. of Math. (2) 48 (1947), 568-640.

[Bu] W. Burnside, Theory of groups of finite order, Cambridge Univ. Press, Cambridge, 1897.

[C1] C. Chevalley, Theory of Lie groups. I, Princeton Univ. Press, Princeton, NJ, 1946.

[C2] _ Theory of Lie groups. II, Hermann, Paris, 1951.

[C3] _ Theory of Lie groups. III, Hermann, Paris, 1955.

[D] M. Duflo, On the Plancherel formula of almost-algebraic real Lie groups, Lecture Notes in Math., vol. 1077, Springer-Verlag, 1984, pp. 101-165.

[GN] I. M. Gelfand and M. A. Naimark, Unitary representations of the classical groups, Trudy Mat. Inst. Steklov. 36 (1950).

[H] Harish-Chandra, Collected papers, 4 volumes, Springer-Verlag, New York, 1984.

[L] R. P. Langlands, Harish-Chandra, Biographical Memoirs of Fellows of the Royal Society 31 (1985), 197-225.

[M1] G. Mackey, Theory of unitary group representations, Univ. of Chicago Press, Chicago, IL, 1976.

[M2] - Induced representations of groups and quantum mechanics, W. A. Benjamin, New York, 1968.

[V] V. S. Varadarajan, Harish-Chandra, Indian Math. Soc. (N.S.) (1991).

[W] E. Wigner, On unitary representations of the inhomogeneous Lorentz group, Ann. of Math. (2) 40 (1939), 149-204.

Department of Mathematics, University of Maryland, College Park, MARYLAND 20742 
